$\underline{\text { Article }}$

\title{
Alkamides and Phenethyl Derivatives from Aristolochia gehrtii
}

\author{
Hosana M. D. Navickiene and Lucia M. X. Lopes" \\ Instituto de Química, Universidade Estadual Paulista, CP 355, 14800-900, Araraquara - SP, Brazil
}

\begin{abstract}
Foram isolados de galhos de Aristolochia gehrtii a cis- $N$-feruloil-3-O-metildopamina, um derivado hemiacetálico do 5-hidroximetilfurfural, e vinte e dois compostos conhecidos. Entre estes compostos estão cinco lignanas, três terpenos, seis alcalóides dos quais cinco são alcamidas, dois derivados do ácido benzóico e seis derivados constituídos pelo grupo feniletil. As estruturas dos compostos isolados foram determinadas por métodos espectroscópicos e comparação de dados físicos e espectroscópicos dos compostos com aqueles disponíveis na literatura.
\end{abstract}

From the stems of Aristolochia gehrtii, the $c i s-N$-feruloyl-3-O-methyldopamine and a hemiacetal derivative of 5-hydroxymethylfurfural were isolated, together with twenty-two known compounds. These compounds include five lignans, three terpenes, six alkaloids (five alkamides), two benzoic acid derivatives, and six phenethyl derivatives. The structures of the isolated compounds were determined by means of spectroscopic methods and comparison with literature data.

Keywords: Aristolochia gehrtii, Aristolochiaceae, $c i s-N$-feruloyl-3-O-methyldopamine, phenylethyl derivatives

\section{Introduction}

As part of our continuing studies ${ }^{1,2}$ on Brazilian Aristolochia species, the constituents of the stems of Aristolochia gehrtii Hoehne were examined. This study led to the isolation of (-)-eudesmin (1), (+)methylpiperitol (2), (-)-hinokinin (4), cubebin (5), and sitosterol (6), whose occurrence is common in the Aristolochiaceae ${ }^{3-6}$. In addition, $\beta$-sitosteryl-D-glucoside (7), isovanillic acid (8), $p$-hydroxybenzoic acid (9) and piperitol (3) were isolated, together with thalipholine (10), 5-hydroxymethylfurfural (11), tyrosol (12), icariside D2 (13), salidroside (14), 3,4-dihydroxyphenethyl alcohol (15), thalictoside (17) and its corresponding aglycone (16). Although aliphatic nitro compounds are unusual in nature $^{7}$, the occurrence of nitrophenanthrene alkaloids is widespread in the Aristolochiaceae ${ }^{8,9}$. In addition, loliolide (18), trans- and cis- $N$-feruloyltyramine (19, 22), trans-and cis- $N$ - $p$-coumaroyltyramine $(\mathbf{2 0}, \mathbf{2 3})$ and trans$\mathrm{N}$-feruloyl-3-O-methyldopamine (21) were isolated. Structural elucidation of the new alkaloid $c i s-N$-feruloyl3-O-methyldopamine (24), isolated for the first time from a natural source, is discussed.

\footnotetext{
*e-mail: lopesxl@iq.unesp.br
}

\section{Experimental}

\section{General}

The NMR spectra were measured on a Bruker and a Varian spectrometers. ${ }^{1} \mathrm{H}$ NMR and ${ }^{1} \mathrm{H}-{ }^{1} \mathrm{H}$ COSY spectra were obtained at 200 and $500 \mathrm{MHz},{ }^{13} \mathrm{C}$ NMR and DEPT were taken at $50 \mathrm{MHz}$. The mass spectra were obtained on an HP5970 spectrometer and on a Fisons Platform II by flow injection into the electrospray source. The instrument was operated in the positive ion mode. The IR spectra were obtained on a Nicolet730 FT-IR spectrometer using $\mathrm{KBr}$ discs. UV absorption was measured in a Hewlett Packard 8452 A diode array spectrophotometer. TLC: Silica gel $60 \mathrm{PF}_{254}$. The purity of the solvents was checked using a Fisons 8060 gas chromatographer coupled to a Fisons VG Platform - column: Supelco, SPB-5 $0.25 \mathrm{~mm}$ i.d. x $30 \mathrm{~m}, 0.25 \mathrm{~mm}$ thickness; oven $100^{\circ} \rightarrow 20^{\circ} \mathrm{min}^{-1} \rightarrow 250^{\circ}$; carrier gas: $\mathrm{He}\left(1.38 \mathrm{~mL} \mathrm{~min}^{-1}\right)$.

\section{Plant material}

The plant material was collected in the Botanical Garden of São Paulo, São Paulo, SP, Brazil, and identified as Aristolochia gehrtii Hoehne by Dr. Condorcet Aranha. A voucher specimen was deposited at the herbarium of the Instituto Agronômico de Campinas, Campinas, SP, Brazil. The material was separated by plant parts, $\operatorname{dried}\left(\sim 45^{\circ}\right)$ and ground. 
<smiles>[R7]c1ccc(C2OCC3C(c4ccc([R9])c([R7])c4)OCC23)cc1[R7]</smiles>

$\begin{array}{llll}\mathrm{R}^{1} & \mathrm{R}^{2} & \mathrm{R}^{3} & \mathrm{R}^{4}\end{array}$

1 OMe OMe OMe OMe

$2 \quad-\mathrm{OCH}_{2} \mathrm{O}-\mathrm{OMe} \quad \mathrm{OMe}$

$3 \quad-\mathrm{OCH}_{2} \mathrm{O}-\mathrm{OH} \quad \mathrm{OMe}$

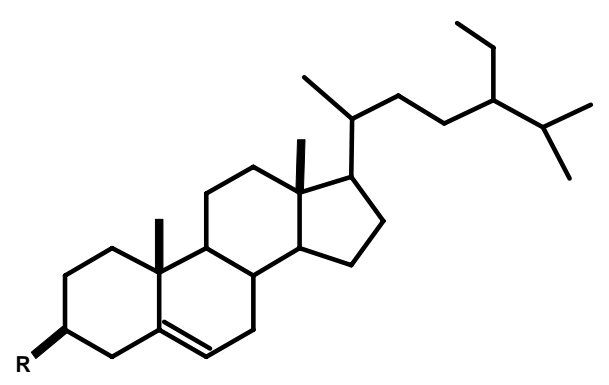

$\mathrm{R}$

6

$\mathrm{OH}$

7 OGlc

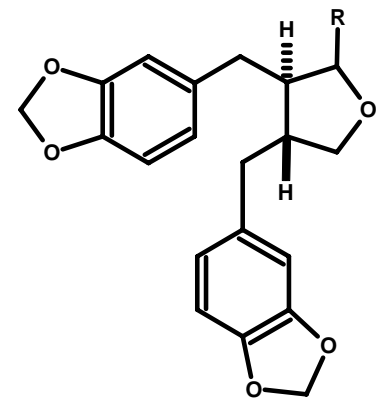

$\mathrm{R}$

$4=0$

$5 \mathrm{OH}$

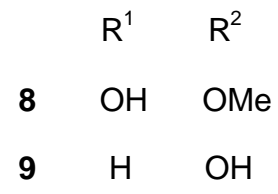

202<smiles>[R]c1ccc(CO)o1</smiles>

$\mathrm{R}$

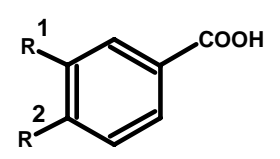

$9 \quad \mathrm{H} \quad \mathrm{OH}$

$11 \mathrm{CHO}$

11a $\mathrm{CH}(\mathrm{OH})(\mathrm{OMe})$ 
<smiles>[2H]CCc1ccc(P)c([TlH])c1</smiles>

$\begin{array}{llll} & \mathrm{R}^{1} & \mathrm{R}^{2} & \mathrm{R}^{3} \\ 12 & \mathrm{H} & \mathrm{OH} & \mathrm{OH} \\ 13 & \mathrm{H} & \mathrm{OGlc} & \mathrm{OH} \\ 14 & \mathrm{H} & \mathrm{OH} & \mathrm{OGlc} \\ 15 & \mathrm{OH} & \mathrm{OH} & \mathrm{OH} \\ 16 & \mathrm{H} & \mathrm{OH} & \mathrm{NO}_{2} \\ 17 & \mathrm{H} & \mathrm{OGlc} & \mathrm{NO}_{2}\end{array}$

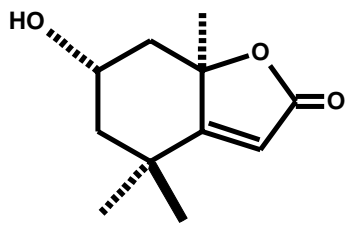

18

\section{Isolation}

Ground stems $(850 \mathrm{~g})$ were extracted exhaustively at room temperature with hexane, $\mathrm{Me}_{2} \mathrm{CO}$ and $\mathrm{EtOH}$ successively and then individually concentrated. The crude acetone extract (4.0 g) was fractionated by CC (silica gel, $80.0 \mathrm{~g}$, hexane-EtOAc gradient) leading to 51 fractions ( $15 \mathrm{~mL}$ ). Frs. 16, 17, 18, 25, $30,35,36,42$, and 43 yielded $\mathbf{6}(16.2 \mathrm{mg}), \mathbf{5}(22.6 \mathrm{mg}), \mathbf{2}$ (86.0 mg), $\mathbf{1}(7.4 \mathrm{mg}), \mathbf{1 8}(9.0 \mathrm{mg}), \mathbf{9}(8.0 \mathrm{mg}), \mathbf{8}(7.7 \mathrm{mg}), \mathbf{7}$ $(67.7 \mathrm{mg})$ and $\mathbf{1 5}(12.2 \mathrm{mg})$, respectively. After prep. TLC [PhMe-MeOH-HOAc (95:5:1)], fr. 12 yielded 4 (3.7 mg).<smiles>[R7]c1ccc(/C=C/C(=O)NCCc2ccc([125I])c([125I])c2)cc1[125I]</smiles>

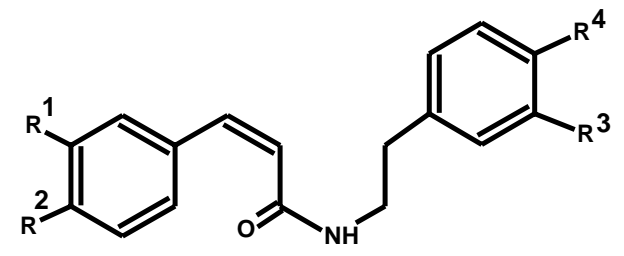

$\begin{array}{lllll} & \mathrm{R}^{1} & \mathrm{R}^{2} & \mathrm{R}^{3} & \mathrm{R}^{4} \\ \mathbf{2 2} & \mathrm{OMe} & \mathrm{OH} & \mathrm{H} & \mathrm{OH} \\ 23 & \mathrm{H} & \mathrm{OH} & \mathrm{H} & \mathrm{OH} \\ 24 & \mathrm{OMe} & \mathrm{OH} & \mathrm{OMe} & \mathrm{OH}\end{array}$

Frs. 15, 21, and 27 by prep. TLC [PhMe-MeOH (95:5)] yielded $\mathbf{1 6}(11,5 \mathrm{mg}), \mathbf{3}(3.0 \mathrm{mg})$ and $\mathbf{1 2}(4.0 \mathrm{mg})$, respectively. Fr. 38 by TLC $\left[\mathrm{CHCl}_{3}-\mathrm{MeOH}-\mathrm{NH}_{4} \mathrm{OH}\right.$ (95:5:1)] afforded $\mathbf{1 9}(18.2 \mathrm{mg}), \mathbf{2 0}(9.4 \mathrm{mg})$ and $\mathbf{2 1}(10.0 \mathrm{mg})$. Frs. 39 and 40 by prep. TLC $\left[\mathrm{CHCl}_{3}-\mathrm{MeOH}-\mathrm{NH}_{4} \mathrm{OH}\right.$ (95:5:1)] yielded mixtures (1:5) of $\mathbf{1 9 + 2 2}(24.2 \mathrm{mg})$ and $\mathbf{2 0}+\mathbf{2 3}(30.8 \mathrm{mg})$, respectively. After TLC [CHCl $-\mathrm{MeOH}$ (93:7)], fr. 41 afforded a mixture (1:4) of $\mathbf{2 1}+\mathbf{2 4}(23.8 \mathrm{mg}$ ). Fr. 45 after prep. TLC $\left[\mathrm{CHCl}_{3}-\mathrm{MeOH}-\mathrm{NH}_{4} \mathrm{OH}\right.$ (80:20:1)] yielded $\mathbf{1 3}$ (13.3 mg), 14 (11.1 mg) and $\mathbf{1 7}(12.0 \mathrm{mg})$.

The ethanol extract of the stems $(18.7 \mathrm{~g})$ was fractionated 
by partition $\left(\mathrm{CHCl}_{3}-\mathrm{H}_{2} \mathrm{O}\right)$. The organic fraction $(855.0 \mathrm{mg})$ was concentrated and dissolved in hot $\mathrm{MeOH}$. After cooling the resulting precipitate was separated and submitted to prep. TLC [PhMe-MeOH (9:1)] leading to the isolation of 11 (8.5 $\mathrm{mg})$ and $11 \mathrm{a}(3.0 \mathrm{mg})$. The aqueous fraction was extracted with EtOAc $(8.5 \mathrm{~g})$. A portion of this fraction $(130.7 \mathrm{mg})$ was submitted to prep. TLC $\left[\mathrm{CHCl}_{3}-\mathrm{MeOH}-\mathrm{NH}_{4} \mathrm{OH}\right.$ (90:10:0.5)] affording 10 (12.5 mg).

$\beta$-sitosteryl-D-glucoside (7)

Colorless solid, mp. $292-297^{\circ} \mathrm{C} \mathrm{Me}_{2} \mathrm{CO}$, decomp., lit. $298{ }^{\circ} \mathrm{C}(\mathrm{EtOH}){ }^{10} \cdot[\alpha]_{\mathrm{D}}-42.0^{\circ}$ (pyridine, $c 2.0$ ), lit. $[\alpha]_{\mathrm{D}^{-}}$ $40.1^{\circ}$ (pyridine, $\left.c 1.3\right)^{10}$. Positive ES-MS $\mathrm{m} / \mathrm{z}$ (rel. int.): $599[\mathrm{M}+\mathrm{Na}]^{+}(100), 577[\mathrm{M}+\mathrm{H}]^{+}(40), 161(35)$.

IR $v_{\max } / \mathrm{cm}: 3423,1595,1485(\mathrm{KBr}) .{ }^{1} \mathrm{HNMR}(500 \mathrm{MHz}$, $\left.\mathrm{C}_{5} \mathrm{D}_{5} \mathrm{~N}\right) \delta 5.40(1 \mathrm{H}, \mathrm{m}, \mathrm{H}-6), 5.08\left(1 \mathrm{H}, \mathrm{d}, J 9.0 \mathrm{~Hz}, \mathrm{H}-1^{\prime}\right), 4.59$ $(1 \mathrm{H}, \mathrm{dd}, J 12.0,2.5 \mathrm{~Hz}, \mathrm{H}-6 '), 4.44(1 \mathrm{H}, \mathrm{dd}, J 12.0,5.3 \mathrm{~Hz}, \mathrm{H}-$ 6'), 4.33 (1H, t, J9.0 Hz, H-3'), 4.30 (1H, t, J9.0 Hz, H-4'), 4.10 $\left(1 \mathrm{H}, \mathrm{t}, J 9.0 \mathrm{~Hz}, \mathrm{H}-2^{\prime}\right), 4.04-3.80$ (2H, m, H-3, H-5'), $2.76(1 \mathrm{H}$, ddd, $J 13.0,5.5,1.5 \mathrm{~Hz}, \mathrm{H}-4), 2.53(1 \mathrm{H}$, br dd, $J 13.0,7.0 \mathrm{~Hz}$, H-4), 2.18 (1H, br d, J 13.0 Hz, H-1), 2.03 (1H, m, H-12), 1.95 (1H, m, H-7), 1.89 (1H, m, H-16), 1.78 (2H, m, H-2, H-20), 1.72 (1H, m, H-25), 1.60 (1H, m, H-16), 1.48-1.24 (8H, m), 1.15 (2H, m, H-12, H-24), 1.04 (1H, m, H-1), 1.03 (1H, d, J 6.5 Hz, H-21), 0.98 (3H, s, H-19), 0.94 (1H, m, H-14), 0.94 (3H, t, $J 7.5$ Hz, H-29), 0.92 (3H, d, J 7.5 Hz, H-27), 0.91 (3H, t, J 7.0 $\mathrm{Hz}, \mathrm{H}-26), 0.71$ (3H, s, H-18). ${ }^{13} \mathrm{C}$ NMR (125 MHz, $\left.\mathrm{C}_{5} \mathrm{D}_{5} \mathrm{~N}\right)$ $\delta\left(\mathrm{C}-1\right.$ to C-29, C-1' to C-6'): $37.2,31.8^{*}, 77.9^{* *}, 39.7,140.7$, 121.7, 31.9*, 50.1, 29.9, 36.6, 21.0, 39.0, 42.7, 56.5, 24.2, 28.2, 56.0, 12.2, 18.9, 36.1, 18.7, 33.9, 26.1, 45.8, 29.2, 19.1, 19.7, 23.1, 12.4, 102.3, 75.0, 78.3, 71.4, 78.1**, 62.5 (Values bearing the same sign may be reversed).

\section{Thalipholine (10)}

Yellow solid, mp. $210-21{ }^{\circ} \mathrm{C}\left(\mathrm{CHCl}_{3}\right)$, lit. $210-211^{\circ} \mathrm{C}$ $\left(\mathrm{CHCl}_{3}\right)^{11}$. IR, UV and ${ }^{1} \mathrm{H}$ NMR: comparable with lit. values ${ }^{11}$. Positive ES-MS $m / z$ (rel. int.): $208[\mathrm{M}+\mathrm{H}]^{+}(100)$, 151 (36). ${ }^{13} \mathrm{C}$ NMR (50 MHz, $\mathrm{CDCl}_{3}$ ) $\delta 190.9$ (C-1), 48.4 (C-3), 27.6(C-4), 108.8 (C-5), 144.6(C-6), 164.9(C-7), 114.2 (C-8), 122.5 (C-9), $132.3(\mathrm{C}-10), 35.2\left(\mathrm{NCH}_{3}\right), 56.0\left(\mathrm{OCH}_{3}\right)$.

\section{Hemiacetal (11a)}

Colorless solid, mp. $35-37{ }^{\circ} \mathrm{C}(\mathrm{MeOH})$. Positive ESMS $m / z$ (rel. int.): $159[\mathrm{M}+\mathrm{H}]^{+}(100), 157\left[^{\mathrm{M}-1]^{+}}(91)\right.$, 143 (24). IR $v_{\text {max }} / \mathrm{cm}: 3470,1522,1384,1025$ (KBr).

\section{cis-N-Coumaroyltyramine (23)}

Yellow solid, $\mathrm{mp} 248-251^{\circ} \mathrm{C}(\mathrm{MeOH}) . \mathrm{IR} v_{\max } / \mathrm{cm}: 3431$, 1718, 1601, 1383 (KBr).
Positive ES-MS m/z (rel. int.): $284[\mathrm{M}+\mathrm{H}]^{+}(69), 306$ $[\mathrm{M}+\mathrm{Na}]^{+}$(100), $322[\mathrm{M}+\mathrm{K}]^{+}(76)$.

${ }^{1} \mathrm{H}$ NMR (200 MHz, CD 3 OD) $\delta 2.65-2.78(2 \mathrm{H}, \mathrm{m}, \mathrm{H}-$ $\left.7^{\prime}\right)$, 3.40-3.49 (2H, m, H-8'), $5.58(1 \mathrm{H}, \mathrm{m}, \mathrm{NH}), 5.79(1 \mathrm{H}$, d, J $12.6 \mathrm{~Hz}, \mathrm{H}-8), 6.60$ (1H, d, J $12.6 \mathrm{~Hz}, \mathrm{H}-7), 6.68$ (2H, d, J 8.5 Hz, H-3, H-5), 6.70 (2H, d, J 8.6 Hz, H-3', H-5'), 7.00 (2H, d, J 8.6 Hz, H-2', H-6'), 7.35 (2H, d, J 8.5 Hz, $\mathrm{H}-2, \mathrm{H}-6) .{ }^{13} \mathrm{C}$ NMR (50 MHz, $\left.\mathrm{CD}_{3} \mathrm{OD}\right) \delta 138.0(\mathrm{C}-7)$, 132.2 (C-2,6), 130.5 (C-2',6'), 121.6 (C-8), 116.7 (C-3,5), 116.0 (C-3', 5'), 42.2 (C-8'), 35.4 (C-7').

\subsection{5. cis-N-Feruloyl-3-O-methyldopamine (24)}

Yellow solid, mp 248-251 ${ }^{\circ} \mathrm{C}(\mathrm{MeOH})$. IR $v_{\max } / \mathrm{cm}$ : 3384, 1718, 1598, 1515, 1271 (KBr). Positive ES-MS $m / z$ (rel. int.): $344[\mathrm{M}+\mathrm{H}]^{+}(57), 366[\mathrm{M}+\mathrm{Na}]^{+}(62), 382$ $[\mathrm{M}+\mathrm{K}]^{+}$(45), 177 (100), 145 (62), 149 (43), 117 (74), 91 (66), 89 (96). ${ }^{1} \mathrm{H}$ NMR (200 MHz, $\left.\mathrm{CD}_{3} \mathrm{OD}\right) \delta$ 2.62-2.67 (2H, m, H-7'), 3.39-3.48 (2H, m, H-8'), 3.79 (3H, s, OMe3'), 3.82 (3H, s, OMe-3), 5.58 (1H, m, NH), $5.80(1 \mathrm{H}, \mathrm{d}, J$ $12.6 \mathrm{~Hz}, \mathrm{H}-8), 6.58$ (1H, d, J $12.6 \mathrm{~Hz}, \mathrm{H}-7), 6.62$ (1H, d, $J$ $8.3 \mathrm{~Hz}, \mathrm{H}-5$ '), 6.65 (1H, d, J 1.6 Hz, H-2'), 6.78 (1H, dd, J 8.3, 1.6 Hz, H-6'), 6.73 (1H, d, J 8.2 Hz, H-5), 6.85 (1H, dd, J 2.0, 8.2 Hz, H-6), 7.45 (1H, d, J 2.0 Hz, H-2).

\section{Results and Discussion}

Extracts from the stems of $A$. gehrtii were fractionated by chromatographic column, followed by preparative TLC, to afford the known lignans $\mathbf{1 - 5}^{4,12-18}$, sitosterol (6) ${ }^{19}$, benzoic acids $\mathbf{8}, \mathbf{9}^{20,21}$, isoquinolone alkaloid $\mathbf{1 0}^{11,22,23}$, phenethyl derivatives 12-17 $7,24-27$, and the terpene $\mathbf{1 8}^{28}$, which were identified by comparison of their physical and spectroscopic (IR, UV, MS, ${ }^{1} \mathrm{H}$ and ${ }^{13} \mathrm{C}$ NMR) data with those reported in the literature. The absolute configurations of lignans $\mathbf{1}$ and $\mathbf{2}$ were established by comparison of their $[\alpha]_{\mathrm{D}}$ values $\left[1:-66.0^{\circ}\left(\mathrm{CHCl}_{3}, c 1.4\right), 2:+74.8^{\circ}\left(\mathrm{CHCl}_{3}, c\right.\right.$ $2.5)]$ with those published in the literature $\left[1:-64.2^{\circ}\right.$ $\left(\mathrm{CHCl}_{3}, c 1.1\right), 2:+76.2^{\circ}\left(\mathrm{CHCl}_{3}, \text { c 2.0) }\right]^{15,16}$. Compound 7 was identified as $\beta$-sitosteryl-D-glucoside by comparison of its ${ }^{1} \mathrm{H}$ and ${ }^{13} \mathrm{C}$ NMR data, as well as by comparison of its melting point ${ }^{10,29}$. From gCOSY, gTOCSY, gHMQC, and $\mathrm{gHMBC}$ experiments, it was possible to assign more feasible $\delta$ values for carbons and hydrogens, including C$3, \mathrm{C}-1$ ' to C-6' and H-3, H-1' to H-6', than those previously described in the literature ${ }^{29}$. The structure was further confirmed by acid hydrolysis, as reported by Ahmed et $a l^{30}$, affording sitosterol and glucose.

Compound $\mathbf{1 1}$ was identified by comparison of its physical and spectroscopic data with those of 5-hydroxymethylfurfural, previously reported ${ }^{21,31}$. It was 
also obtained as a hemiacetal derivative 11a. The ES-MS of 11a showed $[\mathrm{M}+\mathrm{H}]^{+}$at $\mathrm{m} / \mathrm{z} 159(100 \%)$, corresponding to the addition product. The IR spectrum of 11a did not display any carbonyl absorption. The formation of the hemiacetal could be explained by the addition of methanol to 11, since methanol was used as a solvent to solubilize and purify $\mathbf{1 1}$.

Besides three trans-cinnamoylamides 19-21, three pairs of cis- and trans-isomers of cinnamoylamides $(\mathbf{1 9 + 2 2}$, $\mathbf{2 0}+\mathbf{2 3}, \mathbf{2 1}+\mathbf{2 4}$ ) were isolated. Isomers 19-22 were identified by comparison of their spectroscopic data with those previously reported ${ }^{32-35}$. Best separation of the cis isomer 23 from the mixture $(\mathbf{2 0}+\mathbf{2 3})$ was achieved in a $5 / 1$ cis/trans proportion, whereas isomer $\mathbf{2 4}$ from the mixture $(\mathbf{2 1 + 2 4})$ was obtained in a $4 / 1$ proportion. The structures of $\mathbf{2 3}$ and 24 were suggested by their electrospray mass spectra. The ES-MS of 23 displayed an $[\mathrm{M}+\mathrm{Na}]^{+}$at $\mathrm{m} / z 306(100 \%)$, $[\mathrm{M}]^{+}$at $m / z, 284$ (corresponding to $\mathrm{C}_{17} \mathrm{H}_{17} \mathrm{NO}_{3}, 30 \mathrm{~m}$ less than 22), and the ES-MS of 24 displayed an [M] $]^{+}$at $m / z, 344$ (corresponding to $\mathrm{C}_{19} \mathrm{H}_{21} \mathrm{NO}_{5}, 30 \mu$ more than 22), with a base peak at $m / z 177$ (corresponding to a feruloyl moiety). The ${ }^{1} \mathrm{H}$ NMR spectra of both alkaloids showed signals of two cis olefinic hydrogens at $\sim \delta 5.8(1 \mathrm{H}, \mathrm{d}, J 12.6 \mathrm{~Hz})$ and $6.6(1 \mathrm{H}, \mathrm{d}, J 12.6 \mathrm{~Hz})$ assigned to $\mathrm{H}-8$ and $\mathrm{H}-7$, respectively. In addition, two multiplets at $\sim \delta 2.6$ and 3.4 for four methylene hydrogens were observed, which allowed us to establish its cis configuration. Comparison of their IR $\left(v_{C=O}\right.$ : $\left.1718 \mathrm{~cm}^{-1}\right)$ and $\mathrm{UV}\left(\lambda_{\max } 270 \mathrm{~nm}\right)$ spectra with structurally similar alkaloids, such as 22, confirmed the cis configuration at C-7, 8. The main spectroscopic differences observed between the MS, ${ }^{1} \mathrm{H}$ and ${ }^{13} \mathrm{C}$ NMR data of 22 and $\mathbf{2 4}$ were due to the methoxyl group substituent at C-3. The substitution pattern of the aromatic rings was corroborated by ${ }^{1} \mathrm{H}-{ }^{1} \mathrm{H}$ COSY and NOE difference experiments, since they showed the correlations between the methoxyl hydrogens at $\delta 3.79$ and $\delta 3.82$ with the aromatic hydrogens at $\delta 6.65$ and $\delta 7.45$, respectively. The correlations observed between $\mathrm{H}-7$ and $\mathrm{H}-8$ and NH ( $\delta$ 5.52-5.60) confirmed the cis configuration established for 24. Compound 23 had already been obtained from cell cultures of Solarium khasianum, and its TMSi derivative was identified by GC-MS ${ }^{36}$.

To our knowledge, no phytochemical investigation has been carried out on this ornamental species. The occurrence of phenethyl derivatives $\left.\left(\mathrm{C}_{6}-\mathrm{C}_{2}\right) \mathbf{( 1 2 - 1 7}\right)$ is significant in this species. The co-occurrence of biosynthetic derivatives $(\mathbf{1 0}, \mathbf{1 9}-24)$, which could be formed in this species by at least one unit $\mathrm{C}_{6}-\mathrm{C}_{2}$, is remarkable.

\section{Acknowledgements}

The authors thank Fundação de Amparo à Pesquisa do Estado de São Paulo for financial support, including the fellowship to H. M. D. N. and Dr. M. C. M. Young for plant material supply.

\section{References}

1. Bomm, M. D.; Zukerman-Schpector, J.; Lopes, L. M. X. Phytochemistry 1999, 50, 455.

2. Nascimento, I. R.; Lopes, L. M. X. Phytochemistry 1999, 52, 345.

3. Leitão, G. G.; Kaplan, M. A. C. Rev. Bras. Farm. 1992, 73, 65 .

4. Luiz, V.; Bolzani, V. da S.; Trevisan, L. M. V.; Lopes, L. M. X. Quím. Nova 1990, 13, 250.

5. Lopes, L. M. X.; Bolzani, V. da S.; Trevisan, L. M. V. Rev. Latinoamer. Quim. 1988, 19, 113.

6. Lopes, L. M. X.; Bolzani, V. da S. Phytochemistry 1988, 27, 2265.

7. Ina, H.; Iida, H. Chem. Pharm. Bull. 1986, 34, 726.

8. Mix, D. B.; Guinaudeau, H.; Shamma, M. J. Nat. Prod. 1982, 45, 657.

9. Lopes, L. M. X.; Martins, J. A.; Piasentin, R. M. Eclética Quim. 1991, 16, 63.

10. Swift, L. J. J. Am. Chem. Soc. 1952, 74, 1099.

11. Krane, B. D. K.; Shamma, M. J. Nat. Prod. 1982, $45,377$.

12. Oeveren, A.; Jansen, J. F. G. A.; Feringa, B. L. J. Org. Chem. 1994, 59, 5999.

13. Agrawal, P. K.; Thakur, R. S. Magn. Reson. Chem. 1985, 23, 389.

14. Brieskorn, C. H.; Huber, H. Tetrahedron Lett. 1976, 26, 2221.

15. Rao, C. B. S. In Chemistry of Lignan; Andthra University Press, Andhra Pradesh, 1978.

16. Iida, T.; Noro, Y.; Ito, K. Phytochemistry 1983, 22,211 .

17. Honda, T.; Kimura, N.; Sato, S.; Kato, D.; Tominaga, H. J. Chem. Soc., Perkin Trans. I 1994, 1043.

18. Koul, S. K.; Taneja, S. C.; Dhar, K. L.; Atal, C. K. Phytochemistry 1983, 22, 999.

19. Nes, W. D.; Norton, R. A.; Benson, M. Phytochemistry 1992, 31, 805.

20. Pouchert, C. J. In The Aldrich Library of NMR Spectra; vol. 2, Ed. II, Aldrich Chemical Company, Inc, 1985, 195A, 459A.

21. Pouchert, C. J. In The Aldrich Library of IR Spectra; vol. 2, Ed. II, Aldrich Chemical Company, Inc, 1985, 584B, 208B.

22. Chen, I. -S.; Chen, J. -J.; Duh, C. -Y.; Tsai, I. -L.; Chang, C. -T. Planta Med. 1997, 63, 154.

23. Lundstron, J. In The Alkaloids; vol.35, Academic Press; New York, 1989, p.77. 
24. Miyase, T.; Ueno, A.; Takizawa, N.; Kobayashi, H.; Karasawa, H. Chem. Pharm. Bull. 1987, 35, 1109.

25. Miyase, T.; Ueno, A.; Takizawa, N.; Kobayashi, H.; Oguchi, H. Phytochemistry 1989, 28, 3483.

26. Bianco, A.; Naccarato, G.; Passacantilli, P.; Righi, G.; Scarpati, M. L. J. Nat. Prod. 1992, 55, 760.

27. LaLonde, R. T.; Wong, C.; Tsai, A. I. -M. J. Am. Chem. Soc. 1976, 98, 3007.

28. Okada, N.; Shirata, K.; Niwano, M.; Koshino, H.; Uramoto, M. Phytochemistry 1994, 37, 281.

29. Matida, A. K.; Rossi, M. H.; Blumenthal, E. E. A.; Schuquel, I. T. A.; Malheiros, A.; Vidotti, G. J. An. Assoc. Bras. Quím. 1996, 45, 147.
30. Ahmed, W.; Ahmad, Z.; Malik, A. Phytochemistry 1992, 31, 4038 .

31. Hearn, M. T. W. Aust. J. Chem. 1976, 29, 107.

32. Hussain, S. F.; Gözler, B.; Shamma, M.; Gözler, T. Phytochemistry 1982, 21, 2979.

33. Tanaka, H.; Nakamura, T.; Ichino, K.; Ito, K. Phytochemistry 1989, 28, 2516.

34. Muñoz, O.; Piovano, M.; Garbarino, J.; Hellwing, V.; Breitmaier, E. Phytochemistry 1996, 43, 709.

35. Okuyama, T.; Shibata, S.; Hoson, M.; Kawada, T.; Osada, H.; Noguchi, T. Planta Med. 1986, 52, 171.

36. Mühlenbeck, U.; Kortenbusch, A.; Barz, W. Phytochemistry 1996, 42, 1573.

Received: April 19, 2000

Published on the web: April 19, 2001

FAPESP helped in meeting the publication costs of this article. 